\title{
Diskussion
}

\author{
Ferdiansyah Thajib, Francis Seeck, Antke Engel
}

\section{Konflikte umsorgen. Queere Praktiken künstlerischer Kollaboration}

\section{Queere Konfliktkulturen}

Während bis vor kurzem partizipative Kunst der Begriff der Wahl war, ist heute eher von kollaborativer oder kollektiver Kunst die Rede, verknüpft mit der Hoffnung, dass sich dadurch solidarische Verbindungen stärken (Awad et al. 2019) und Diskriminierung anfechten ließen (Husse in Lang 2019). ${ }^{1}$ Partizipation suggeriert, dass jemand den Rahmen setzt und andere zur Teilnahme einlädt, wie dies bei Projekten kultureller Bildung üblicherweise der Fall ist. Konflikte sind als Autoritätskonflikte und durch strukturelle Asymmetrien ${ }^{2}$ vorprogrammiert. Doch das angeblich demokratischere Setting der kollektiven Kollaboration ist unserer Ansicht nach nicht weniger konfliktträchtig, wird in Betracht gezogen, dass neben strukturellen Asymmetrien auch ein Spektrum sozialer Ungleichheiten zum Tragen kommen kann - zumindest dann, wenn eine heterogene Gruppe versucht zusammenzuarbeiten. Uns interessiert deshalb, ob und wie in künstlerischen Projekten mittels künstlerischer Praxen auf Konflikte reagiert werden kann und was diesbezüglich ein queer/feministisches und intersektionales Selbstverständnis beizutragen hat. Wir möchten mit der These beginnen, dass es queerer Konfliktkulturen bedarf, um diskriminierende und (selbst-)ausbeuterische Momente partizipativer, kollaborativer oder kollektiver Projekte zu verändern. 'Queere Konfliktkulturen ist ein Arbeitsbegriff, den wir dem Konzept von Caring for Conflict (CfC) entnehmen, einem Projekt kultureller Bildung, an dem wir beteiligt waren und über das wir hier reflektieren, um theoretisch-konzeptionelle Einsichten zu gewinnen:

1 Bacharach / Booth / Fjaerstad (2016) sehen Kollaboration als Effekt eines zunehmend kommerzialisierten Kunstfeldes.

2 Z.B. wer hat die Definitionsmacht über das Konzept, wer hat die Leitungsfunktion, kann über Gelder verfügen, hat eine bezahlte Stelle, kann solche vergeben? 
> Queere Konfliktkulturen sind ein Plädoyer dafür, Lust an Komplexität und Irritation zu entwickeln und zugleich Konflikte als Chance für Veränderung aufzugreifen. Indem Konflikte als Machtkämpfe angesehen werden, kann auch die Aufmerksamkeit auf Strukturen der Ungleichheit und Geschichten der Gewalt gelenkt werden, die ihre Dynamik antreiben. Ist es möglich, in Konflikten ein utopisches Potenzial zu entdecken, das deren Bewegung in Richtung Gerechtigkeit antreibt? (Institut für Queer Theorie o.J.)

Der Begriff dient nicht der Zustandsbeschreibung, sondern der Spekulation über künftige Möglichkeiten, die es im Zuge eskalierender Spannungen und Verwirrungen noch nicht gibt. Diese künftigen Möglichkeiten, so unsere These, entfalten sich mittels dessen, was wir 'Konflikte umsorgen nennen. Konflikte sind in diesem Sinne nicht als Problem, sondern als Hinweis auf und Einladung zur Veränderung von Machtverhältnissen zu verstehen (Reitmair-Juárez 2016). Sie zu umsorgen, statt sie zuzuspitzen, abzuwehren oder durch eine harmonisierende, kompromissfreudige Lösung stillzustellen, lenkt die Aufmerksamkeit so lange auf bestehende Machtungleichgewichte sowie Diskriminierungs- und Gewaltverhältnisse, bis diese tatsächlich abgebaut sind. Das Umsorgen verweist hierbei zum einen auf einen feministischen Care-Begriff (von Engl. care), der ausgehend von einem Wissen um Relationalität und Angewiesenheit die Arbeit betont, die im Umsorgen liegt, ebenso wie die Risiken von Ausbeutung und Abhängigkeit (vgl. Puig de la Bellacasa 2011; Tronto 2013; Conradi 2016). Im Sinne eines queeren Care-Begriffes möchten wir darüber hinaus die messiness hervorheben, die Sorgeverhältnissen innewohnt. Der Kulturanthropologe und Queer Theoretiker Martin Manalansan (2018) argumentiert dafür, Care-Praktiken und Beziehungen als messy, ungeordnet, ambivalent und widersprüchlich zu verstehen. Ausgehend von einer ethnographischen Studie zu Überlebensstrategien einkommensarmer queerer Migrant $\star^{i}$ nnen in New York zeigt er, wie auch ein gegenseitiges Ignorieren und andere Praktiken, die aus normativer Perspektive als uncaring gelabelt werden, eine Form von Care darstellen können (ebd.). Zudem entstehen aus diesen Praxen Formen >queerer Sozialität (Povinelli 2011) oder scommunities of care (Laufenberg 2012), die das Geprägtsein dieser Gemeinschaften durch strukturelle Gewalt (z.B. der Migrationsregime) nicht leugnet, sondern als Bedingung der Beziehungsgefüge ernst nimmt.

Der Begriff queer in squeere Konfliktkulturen besagt also, dass eine normative Ausrichtung von Zusammenarbeit als harmonisch und konsensuell verweigert wird, und zwar weil dies der Veränderung von Machtun- 
gleichgewichten nicht dienlich ist. Stattdessen wird darauf gesetzt, mit der paradoxen Spannung zwischen Konflikt und Care zu experimentieren. Wie verknüpft sich dies nun mit künstlerischen Praxen und ästhetischen Fragestellungen? Bevor das Hauptthema dieses Aufsatzes anhand von zwei Fallstudien der Teilprojekte von Caring for Conflict (CfC) erörtert wird, bietet der folgende Abschnitt eine Einführung in das Verständnis kollaborativer künstlerischer Praxis. CfC wird hier eingeordnet und unsere jeweiligen Rollen in verschiedenen Phasen des Projekts werden erläutert.

\section{Kunst, Kollaboration und Konflikt}

Kollaboration hat sich zu einer etablierten Arbeitsweise in verschiedenen Bereichen zeitgenössischer europäischer und anglo-amerikanischer Kunstpraxis entwickelt. Dieser Begriff - manchmal auch als Kooperation, Partizipation, Interaktion oder kollektive Aktion benannt - umfasst Methoden, die mehr als eine $\star_{n}$ Akteur $\star_{i n}$ einbeziehen und wahlweise prozess- oder produktorientiert ausgerichtet sein können (Lind 2009, 54). Obgleich in unterschiedlichen Formen kollaborativ künstlerisch gearbeitet wird, ist dies doch immer mit dem Anliegen verbunden, sich der Kommodifizierung von Kunst durch Demokratisierung der künstlerischen Produktion zu widersetzen. Eine solche Orientierung treibt kollaborative Kunstpraktiken in eine ständige Auseinandersetzung mit Spannungen zwischen kollektiver und individueller Autor ${ }^{\star}$ innenschaft sowie den damit einhergehenden ethischen und politischen Werten und daraus resultierenden ästhetischen Entscheidungen (Bishop 2012). Anstatt diesen Prozess als egalitäre und harmonische Begegnungen in der Differenz zu charakterisieren, stehen konflikthafte, wenn nicht agonistische Aushandlungen und Inszenierungen der Zusammenarbeit im Vordergrund. Diese Dimension ist noch ausgeprägter, wenn wir uns die Funktionsweise der Zusammenarbeit in queer/feministischen künstlerischen Praktiken ansehen. Amy Tobin (2019) zeigt, wie der Einsatz queer/feministischer künstlerischer Methoden zur Destabilisierung normativer und patriarchalischer Strukturen weit davon entfernt ist, Gruppenkonformität, Kompromisse und Konsens zu erzielen. Insofern diese Methoden auf Umverteilung der Macht zwischen Künstler`innen, Kunstinstitutionen und der Öffentlichkeit zielen, sind sie gezwungen, mit Ungleichheiten, Unterdrückung und Ausschlüssen aufgrund von Rassisierung, Klasse und Geschlecht - und den entsprechenden Widerständen auch innerhalb der Projekte - umzugehen. 
CfC, eine Kooperation vom Kunstraum District und dem Institut für

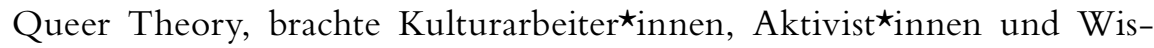
senschaftler ${ }^{\star}$ innen zusammen, die ihre Praxis als queer/feministisch und intersektional verstehen. Gemeinsam mit Jugendlichen aus Schulen, Mädchen $\star_{-}$und Jugendzentren wurden künstlerische Projekte entwickelt, die anhand von Themenfeldern wie z.B. Wem gehört die Stadt?, sprachliche Gewalt/sprachliche Ermächtigung oder vergeschlechtlichte Körperpraxen Aufmerksamkeit auf komplex verflochtene Differenzen, Mehrfachzugehörigkeiten und Machtverhältnisse lenkten. Das zweijährige Projekt (2017-2019) bestand aus verschiedenen Komponenten kollaborativer Kunstpraxis, von denen zwei im Folgenden diskutiert werden. Wir Autor ${ }^{\star}$ innen waren in unterschiedlichen Rollen und Kapazitäten beteiligt. Antke Engel und Ferdiansyah Thajib gehörten zu denjenigen, die zusammen mit Suza Husse von District die erste Idee für dieses Projekt hatten. Es hieß 2016 noch `Zusammenhalt im Konflikt und startete mit der These, dass es angesichts der Heterogenität heutiger Gesellschaften und diverser Krisenerfahrungen vielversprechend sei, queere Formen im Umgang mit Konflikten auszuloten und produktiv zu machen. Im Förderantrag für den Berliner Projektfonds Kulturelle Bildung wurde der Begriff Caring for Conflict geprägt und die Forschungsfrage gestellt, was >queere Konfliktkulturen sein könnten und inwiefern diese mittels künstlerisch-kultureller Praxen zu fördern seien. ${ }^{3}$ Francis Seeck ist im zweiten Projektjahr $(2018 / 19)$ eingestiegen und hat gemeinsam mit Thajib die so genannte Arena moderiert, eine Workshop-Reihe, die als Plattform der kollektiven Reflexion zwischen sämtlichen Teilprojekten geschaffen wurde. Im Folgenden werden wir argumentieren, dass die Arena nicht nur die verschiedenen künstlerischen Initiativen von CfC in Austausch miteinander gebracht hat, sondern ihrerseits eine künstlerische Praxis der kollaborativen Formierung von queeren Konfliktkulturen war.

Dass CfC, ebenso wie dieser Artikel, sich somit im Grenzbereich zwischen Kunst, Wissenschaft, künstlerischer Forschung und Pädagogik bewegt, erscheint uns queer / feministisch produktiv. Konzeptionelle Reflexion wird somit nicht in die Wissenschaft verlagert und aus Kunst und kultureller Bildung herausgehalten, sondern als zentraler Bestandteil ange-

3 Beantragt und im ersten Projektjahr geleitet durch Antke Engel, Suza Husse, Nino Hälcker und Andrea Keppler. Engel hat zudem im ersten Projektjahr ein Salonformat angeboten; Thajib war 2017/18 einer der beteiligten Künstler^innen des Teilprojekts Geheimsprachen/Gemeinsprachen, das Workshops mit Jugendliche durchführte, in denen mit (Körper-) Sprache als Modus der Konfliktbearbeitung in alltäglichen Interaktionen experimentiert wurde. 
sehen. Durch die konzeptionelle Arbeit am Begriff 'Konflikte umsorgen wird die bewährte feministische Kritik an der Trennung von Öffentlichem, Privatem und Persönlichem reaktiviert, aber auch die für Care-Praxen typische Aufmerksamkeit für Beziehungen der Angewiesenheit, der körperlichen und emotionalen Bindungen als Grundelementen der Sozialität in die Praxen von Kunst und Wissenschaft einbezogen. Insbesondere für kollaborative Projekte kann ein solches Interesse an den emotionalaffektiven Dimensionen von Beziehung aus intersektional queer/feministischer Perspektive auch heißen, dass heteronormative, rassistische, klassistische und ableistische Formatierungen reflektiert werden. Die Suche nach Alternativen kann sich dann aber nicht auf die Kritik an stereotypen Geschlechterbildern, binären Geschlechterlogiken, reproduktiver Zeitlichkeit und komplementären Parbeziehungen beschränken, sondern muss wahrnehmen, welche Spannungen und Konflikte entstehen, wenn z.B. Zeitlichkeit zugleich auch als rassistische Fortschritts- und ableistische Effizienznorm daherkommt, wenn Paar- und Familienformen bürgerlichweiße Überlegenheitsvorstellungen reproduzieren oder Geschlechterstereotype sich als milieu- und kontextspezifisch erweisen. Derartige, intersektional bedingte Konfliktpotenziale aufzugreifen, ist in der Regel nicht das, was in etablierten Kontexten von Kunst, Wissenschaft und Pädagogik auf der Tagesordnung steht. Was also kann es heißen, mittels künstlerischer Praxen und ästhetischer Formgebung das Potenzial von Konflikten $\mathrm{zu}$ aktivieren, in Machtgefüge und -ungleichheiten verändernd einzugreifen (Diendorfer 2016)? Wenn es also nicht nur darum geht, Streit, Meinungsverschiedenheiten sowie Kämpfe um Ressourcen und Anerkennung auszutragen, sondern dabei Machtungleichgewichte abzubauen, kommt dann kollaborativen künstlerischen Praxen im Hinblick auf derart machtund herrschaftskritische Transformationen eine besondere Bedeutung zu? Oder schwingt auch in dieser Frage erneut ein bürgerlich-elitäres Kunstverständnis mit, das die materiellen Möglichkeitsbedingungen der Kunstproduktion bzw. der existentiellen Prekarität im Falle deren Fehlens unterschätzt? Auch wenn unter der Überschrift der Kollaboration die Idee eines einzigartigen Schöpfergenies verabschiedet wird, bleibt doch das Kreativitätsparadigma bestehen und wird die Erfüllung im schöpferischen Tun höher geschätzt als die Frage der Asymmetrien und Machtinteressen, die die Zusammenarbeit prägen. Deshalb möchten wir im Rahmen dieses Beitrags zugespitzt fragen, wie im Kontext der kollaborativen Praxen von CfC mit sozialer Heterogenität und den daraus resultierenden Konflik- 
ten umgegangen wird. ${ }^{4}$ Finden Konflikte im Konzept der Kollaboration einen systematischen Ort? Wird Kollaboration - gerade in feministischen Kontexten - tendenziell zu harmonisch gedacht? Oder können Feminismen im Gegenteil auf eine lange Tradition des Streits zurückgreifen, von der auch queere Konfliktkulturen profitieren?

\section{Queere künstlerische Konfliktpraktiken}

\section{Streitbauten - wie Architektur Begegnungen formt}

Das Kunst- und Architekturprojekt sStreitbauten surde im Rahmen von CfC unter der künstlerischen Leitung von Ä̈cha Diallo und Lorenzo Sandoval durchgeführt. Ausgehandelt wurden mit Schüler`innen und Jugendlichen des Treffpunktes Fresh 30 die Fragen: Welche Konflikte und Ausschlüsse werden in urbanen Räumen geschaffen und wie werden diese Konflikte verhandelt? In der Streitbauten-Werkstatt bauten sie zusammen eine Kollektivskulptur. Sie schufen einen gemeinsamen Raum mit spitzen Ecken, sanften Nischen, wackelnden Säulen und wehenden Flächen. Sie beschäftigten sich mit den Fragen: "Wie fangen wir an? Welches Teil kommt wo hin? Was hält sich woran fest oder stößt sich ab? Wo und wie findet jede ${ }^{\star}$ r einen Platz im Gemenge? Jedes neue Modul verändert das kollektive Raumgefüge (District 2017). Sie setzen sich mit Materialien und Formen auseinander. Diese »transportieren Spannung und Gegenspannung, Gewimmel und Kollaps, Hartes und Weiches, Unbewegliches und Elastisches, Vertikales und Horizontales« (ebd.).

KLIRRRRR Festival hatten wir die Festivals betitelt, mit denen jeweils die Projektjahre abgeschlossen wurden. Ein lautmalerischer Titel, der keine Illusionen aufkommen lässt, dass umsorgte Konflikte nichtsdestotrotz in einem Haufen Scherben enden können - ob das klingende Klirrrrren jedoch ein befreiendes, ein aggressives, ein zelebrierendes, ein spielerisches, kultiviert wild oder wilder Kult ist, war damit keineswegs ausgemacht (vgl. District 2018).

$\mathrm{Zu}$ erwähnen ist jedoch, dass für das Festival nicht die Kollektivskulptur installiert wurde, an der die Jugendlichen gebaut hatten. Stattdessen benutzte District für das Festival einen architektonischen Entwurf, der von

4 Im Unterschied zu den Begriffen Vielfalt oder Diversität beinhaltet der Begriff Heterogenität den Hinweis auf die Verschiedenartigkeit und die unterschiedlichen Entstehungsweisen seiner Komponenten. Der Begriff findet insbesondere in der Pädagogik und den Bildungswissenschaften Anwendung (Walgenbach 2017). 
einem der teilnehmenden Künstler, Lorenzo Sandoval, geschaffen wurde. Hängt mit dieser Perfektionierung auch zusammen, dass die Streitbauten während der beiden KLIRRRRR-Festivals, bei denen sie in der Ausstellungshalle zum Gebrauch einluden, gar nicht zum Austragen von Streit oder Konflikten genutzt worden sind? Sie dienten eher zur Exploration und Reflexion, welche als dezidiert gemeinschaftliche Praxen, z.B. in Form einer Teezeremonie, inszeniert wurden. Da diese jedoch nicht in Konflikte mündeten, lässt sich aus ihnen auch schwerlich ein Beitrag zu queeren Konfliktkulturen extrahieren. Ähnliches gilt für die imaginären Reisen auf Landkarten, die die dekoloniale Feministin Gloria Anzaldúa gezeichnet hatte, und die von der Künstlerin Verena Melgarejo Weinandt für einen Workshop mitgebracht wurden. Sie erlaubten, das Leben von Anzaldúa in den von ihr so genannten borderlands zwischen Geschlechtern und Ethnisierungen sowie den USA und Mexiko nachzuvollziehen und sich selbst in Verhältnis zu setzen. Doch die entsprechende Erfahrung asymmetrischer und spannungsreicher oder auch identifikatorischer Relationen setzten sich in keine Streit-Nutzung der Streitbauten um. Statt spontan mit dem Raum zu experimentieren, folgten die Teilnehmenden den Vorgaben der Workshopleitung. Doch warum wurden die angepeilten Nutzungsweisen von den Teilnehmenden einfach angenommen? Warum wurde kein Widerstand geleistet?

Die Streitbauten, obwohl architektonisch so konzipiert, dass sie zur Konfliktaustragung taugen, haben also eher strukturelle und kulturelle als relationale Dimensionen des Konflikts in den Blick genommen. Waren es die warmen Farben, die Leichtbauweise aus Stoffbahnen, die potenzielle Mobilität und Veränderbarkeit des temporären Raumes, die dazu eingeladen haben, eher zur Ruhe zu kommen als zu streiten? Wann bedeutet dies ein Ausweichen? Wann ermöglicht es, den Blick weiter schweifen zu lassen, über die unmittelbaren Spannungen hinaus hin zu den Mustern und Bedingungen und Vorgeschichten eines Konflikts?

Im zweiten Jahr wurde eine spezifische Streitarchitektur, die Arena, erprobt, ohne dass zunächst ein expliziter Zusammenhang zum Teilprojekt der Streitbauten hergestellt wurde. Uns erscheint dies im Nachhinein jedoch durchaus sinnvoll, weil für uns deutlich wurde, dass die materielle (architektonische) Form wichtig ist, sie aber keinen Ersatz dafür darstellt, die kommunikativen Prozesse zu moderieren. Diese als Machtprozesse zu verstehen, in denen affektive und Beziehungsdynamiken, persönliche und kollektive Geschichten ebenso wie materielle und strukturelle Bedingungen zum Tragen kommen, mittels derer Diskriminierungen und Privilegien verteilt werden, war Anliegen der Arena. 


\section{Care in der Arena - die Arena, eine Streitarchitektur}

Die Begriffswahl `Caring for Conflicts/Konflikte umsorgen`soll das transformative Potenzial von Konflikten andeuten, bringt allerdings auch irreleitende Assoziationen des Umgehens von Machtdynamiken und der Überwindbarkeit von Konflikten hervor. Manche Teilnehmer^innen sahen darin ein Versprechen auf ausreichend Sorge für alle, um dann festzustellen, dass sie auf ein illusorisches anders gehofft hatten. Die Arena hingegen rief in ihrer Begrifflichkeit einen Kampf- oder zumindest kompetitiven Raum auf, erwies sich dann aber in der Umsetzung als einladend, eine Vielfalt von Perspektiven nicht nur wahrzunehmen, sondern auch in Auseinandersetzung miteinander zu erleben.

Eine Arena ist ein Raum, in dem die Frage »Wer setzt sich durch?» im Mittelpunkt schwebt. Es ist üblicherweise eine cis-männliche Kampfarena, ausgerichtet auf Gewinn und mit einer sportlichen Komponente versehen, auch wenn es um wirtschaftlichen oder militärischen Erfolg, um Leben oder Tod geht. Care-Arbeit bleibt hier in der Regel unsichtbar und findet erst nach dem Kampf statt: Verletzte Lebewesen werden nach dem Ende der Show zusammengeflickt. Sich in eine Arena zu begeben ist riskant. Manche suchen das Risiko; andere vermeiden es, weil sie nicht zerrissen werden wollen.

Für Seeck und Thajib, die das Workshop-Format der Arena ausarbeiteten, stellte sich die Frage, wie sich innerhalb der Arena, also in Anerkennung dessen, dass sich ohne Konflikte nichts ändern wird, Streit und Care miteinander verbinden lassen. Wie können wir Care-Arbeit in die Arena verlegen? Und wie können wir übliche Arbeitsteilungen, die sich im Feld des Umsorgens ausbilden, machtkritisch umbauen? Zu diesem Zwecke wurden individuelle Reflexions- mit gemeinsamen Gesprächsformen verknüpft. Es wurde Erinnerungsarbeit geleistet, bei der die Beteiligten ihre Care-Biografien und aktuelle Eingebundenheit in Care-Beziehungen rekonstruierten und miteinander teilten. Dies schuf eine neue, breitere Grundlage, um Konflikte zu verstehen, die sich innerhalb von CfC rund um Fragen der Definitionsmacht, der Verantwortung, der Ressourcenverteilung, der finanziellen und kommunikativen Wertschätzung, um Entscheidungsspielräume und Weisungsgebundenheit entwickelt und bei allen - wenngleich unterschiedliche Formen der - Erschöpfung bewirkt hatten. Wir fragten uns: Wälzen wir unsere Erschöpfung auf andere ab? Und können wir dies durch veränderte Praktiken und Kommunikationsformen ändern? Oder stoßen wir auf strukturelle Hierarchien, Diskrimi- 
nierungen, Privilegien und Ressourcenmängel, die sich nicht ausgleichen, sondern nur zurückweisen lassen?

Wir waren uns einig: Wer welche Care-Arbeit verrichtet und erhält, ist eine vergeschlechtlichte, rassifizierte und eine Frage von Klasse. Wir erzählten uns, wer in unseren Herkunftsfamilien oder Institutionen, in denen wir aufgewachsen sind, Care-Arbeit geleistet hat. Unter dem Begriff diaspora care machte Nuray Demir, Künstlerin und in der Projektleitung der zweiten CfC-Phase, darauf aufmerksam, dass sie als Kind bereits ihre Eltern zu Elternabenden und Ärzt $\star^{\star}$ innen begleitete, um zu übersetzen. Care ist immer auch ein intersektionales Thema: Wer kann sich ausruhen und hat ein Sicherheitsnetz, und wer ist getrieben? Übersetzt für CfC heißt dies: Care wird oft als Norm oder Aufforderung formuliert: Wir sollen uns mit dem Projekt identifizieren und Überstunden gerne leisten, da wir uns ja für das Thema sorgen. Gleichzeitig soll gerade bei Kunstprojekten am Ende ein Produkt stehen, welches für die Förder`innen sichtbar ist. Trotz kurzer Laufzeiten besteht die Erwartung, etwas Ausgereiftes zu produzieren oder die Beteiligten, besser noch, eine Öffentlichkeit fundamental aufzuwirbeln und zu verändern. Typisch ist auch, dass kollektive Arbeits- und Denkprozesse am Ende unsichtbar werden, wenn Artikel oder künstlerische Arbeiten unter Einzelnamen veröffentlicht werden. Andererseits ist auch die Nichtnennung von Namen und die Unterordnung unter ein Projekt ein Problem. Gerade in der kulturellen Bildung, der eine Hierarchie strukturell eingeschrieben ist, bedeutet dies, dass diejenigen auf der Empfängerseite oft funktionalisiert werden. So generieren z.B. Kinder und Jugendliche durch spielerischen, performativen Einsatz oder durch Geschichtenerzählen Inhalte und kreative Formen, die von beteiligten Künstler^innen oder Wissenschaftler ${ }^{\star}$ innen in Eigenregie (neu) zu Kunst-Objekten verpackt oder in wissenschaftliches Wissen übersetzt werden.

Kollaborativen Kunstprojekten, so ein Ergebnis der Arena-Diskussionen, muss es also gelingen, diese strukturellen Hierarchien und die Arbeitsteilung nach professionalisierten Rollen (Kurator ${ }^{\star}$ innen, Orga/AdminTeam, Forscher^innen, Künstler^innen, Projektteilnehmende) anfechtbar zu machen. Nur dann, und hier wird es brisant, können auch Konflikte, die durch soziale Ungleichheit, durch diverse -ismen und durch asymmetrische Angewiesenheit genährt sind, ausgetragen werden. Konflikte umsorgen heißt also, materielle und kommunikative Räume zu schaffen, in denen die Care-Beziehungen des eigenen Projekts zur Verhandlung stehen: Wer trägt was bei? Wer profitiert wovon? Wer geht sorgsam mit welcher Art Konflikte um? Mit Konflikten, die vielleicht überhaupt erst 
entstehen, weil jemand das eigene Privileg ausspielt, gesund, weiß, finanzkräftig, gebildet, sozialversichert, verstaatsbürgert, heterosexuell und / oder cis zu sein? Probeweise schlagen wir vor: Konflikte umsorgen heißt, Konflikthaftigkeit wahrnehmen, Machtdynamiken untersuchen und unterbrechen sowie Konsequenzen ziehen, die Machtungleichgewichte abbauen.

Bleibt die Frage: Was erlaubt es, die Arena als Streitarchitektur und als künstlerisches Format zu verstehen? Für CfC wurde die runde Form der historischen Arena beibehalten. Die Gespräche fanden in verschiedenen Kreisformationen statt, in denen teils in Paaren, teils in Kleingruppen, teils im Plenum nachgedacht, geschrieben/gezeichnet oder gesprochen wurde. Entscheidend war: Die Konstellationen haben gewechselt, ohne dass Positionen von Gewinnenden und Verlierenden festgeschrieben wurden. Entscheidend war: Es gab kein Publikum, sondern die `Tribünen` waren immer auch Orte der Auseinandersetzung. Der Innenraum der Kreise blieb teilweise leer; er war also kein Zentrum eines Spektakels, dem passiv beizuwohnen war. Manchmal war er jedoch mit Objekten oder Begriffen bestückt (von Teilnehmenden mitgebracht, von den Organisator`innen gestellt), die als Werkzeuge (funktional) oder Fetische (lustvoll) aufgegriffen und in die Zirkulation gebracht werden konnten. Manchmal war der Innenkreis auch Bewegungsraum, in dem sich Choreographien oder spontane Bewegungsnetze entfalten konnten, mittels derer die beteiligten Körper sich ins Verhältnis zueinander setzen und Nähe, Distanz, Begegnung oder Trennung inszenieren konnten.

Wir verstehen dies als künstlerische Praxis, insofern hier durch Formgebung und Gestaltung Situationen sinnlicher Erfahrung geschaffen werden. Deren Potenzial zur Veränderung von Machtverhältnissen liegt unserer Ansicht nach darin, dass in der künstlerisch gestalteten Situation sinnliche Wahrnehmung, verkörperte Geschichte und Befähigungen, affektive Verwicklung, Vermittlung von Inhalten, Erkenntnis sowie Reflexion und Interpretation gleichermaßen bedeutsam sind und sich gegenseitig stärken, statt einander auszuschließen. Zudem lebt Kunst von der Spannung zwischen Produktions- und Rezeptionserfahrungen, die sich weder zur einen noch zur anderen Seite hin auflösen lässt. Darüber hinaus verbinden sich in kollaborativen Prozessen individuelle und geteilte Dimensionen, ohne dass sie ineinander aufgehen. Somit bewirkt (kollaborative) Kunst eine Komplexität, die in den üblichen Formen pädagogischer oder politischer Konfliktbearbeitung nicht erreicht wird. 


\section{Was kann werden? - dynamische Formen ohne Zentrum}

Caring for Conflict (CfC) verdeutlicht, wie das Umsorgen von Konflikten durch künstlerisch-kollaborative Praxen im wahrsten Sinne des Wortes >Form annimmt . Im Falle der Streitbauten und deren Installation während des KLIRRRRR-Festivals wurde das Potenzial architektonischer Interventionen ausgelotet. Diese versuchen, Spannungen zwischen privatem Streit und öffentlichem Konflikt einen Raum zu geben und es zeigt sich, wie ein sinnlich-aufmerksames Wandern es ermöglicht, die Spannungen zu manövrieren, wenn die Form keine Vorschriften erlässt. Allerdings bewirkte die sgebaute Absicht auch, dass sich Konflikte nicht zuspitzten, sondern eher vage blieben und die Erforschung historischer Konfliktmuster nicht zur Übertragung ins Heute einlud. Auch in anderen Teilprojekten von CfC stellten wir fest, dass dann, wenn konkrete Konflikte primär als Effekt struktureller Probleme (Stadtpolitik, Migrationsregime, Rassismus in Medien und Curricula) verstanden wurden, sich aufgrund der etablierten Symmetrien kaum Möglichkeiten der Konfliktaustragung auftaten. Hingegen lenken biographische und narrative Strategien die Aufmerksamkeit darauf, wie inmitten der Unordnung des Lebens alternative Formen von Care auch mit Formen der Konfliktbearbeitung einhergehen. Nicht zuletzt die Fallstudie der Arena verdeutlicht, dass im Konflikte Umsorgen Unvereinbarkeiten zwischen verschiedenen Positionierungen zugleich anerkannt und als Anlass für Veränderung verstanden werden können. Die Dynamiken der Arena zeigen Wege auf, wie kollektiv mit struktureller Prekarität so umgegangen werden kann, dass individuelle Bedürfnisse unterstützt werden. Konfigurationen von Macht, Privilegien, Vergnügen und Verantwortung im Zusammenhang von Care und Kunst werden von einem einzelnen individuellen oder institutionellen Akteur auf Formen ohne Zentrum oder mit vielen Zentren verlagert.

Der Nachhall einer solchen Beharrlichkeit, der sich eher für Auswirkungen von Prozessen als für Ergebnisse von Produktionen interessiert, lässt sich unserer Ansicht nach an den organisatorischen Veränderungen erkennen, die in letzter Zeit innerhalb von District stattgefunden haben. Mitte 2019, etwa zeitgleich mit dem Ende von CfC, änderte dieser Kunstraum seinen Namen in District * Schule ohne Zentrum. Dieser Schritt war mehr als eine Umbenennung. Vielmehr ging er mit der Überarbeitung der Organisationsstruktur, des Programmformats, der kuratorischen Ausrichtung und dem Übergang von einer $\mathrm{GmbH}$ in einen eingetragenen Verein einher. Verantwortlichkeiten, auch die kuratorischen, wurden auf vielfältigere Akteur^innen verteilt. 
Wir sehen eine starke Resonanz zwischen den Einsichten, die während der Aktivitäten im Rahmen von CfC gewonnen wurden, und der kürzlich erfolgten Transformation von District insbesondere durch die offene Einladung, sich mit Brüchen und Veränderungen als Modi der Förderung einer queer / feministischen und dekolonialen Zukunft auseinanderzusetzen. Die institutionellen Umstrukturierungen bewirken, dass Kollaboration zu einer Lebens- und Arbeitsweise wird, in der Machtungleichheiten problematisiert werden können und die Reproduktion struktureller Gewalt nicht unbemerkt bleibt. Dies ist ein Beispiel, wie der Anspruch auf Dissonanz und das Verbleiben in der Konfusion, die durch CfC konzipiert und gefördert werden, über den Rahmen eines künstlerischen Kollaborationsprojekts hinaus aktiviert werden können.

\section{Literatur}

Awad, Juana et. al.: teilen / lernen. Methoden des Kollektiven in Kunst, Wissenschaft und Aktivismus. (2019). www.udk-berlin.de/forschung/temporaere-forschungseinrichtun gen/dfg-graduiertenkolleg-das-wissen-der-kuenste/veranstaltungsarchiv-des-dfg-gra duiertenkollegs/teilenlernen-methoden-des-kollektiven-in-kunst-wissenschaft-undaktivismus/ [Stand: 15.07.2020].

Bacharach, Sondra / Booth, Jeremy Neil/Fjærestad, Siv B. (Hrsg.) (2016): Collaborative art in the twenty-first century. London.

Bishop, Claire (2012): Artificial Hells. Participatory Art and the Politics of Spectatorship. London / Brooklyn.

Conradi, Elisabeth (2016): Die Ethik der Achtsamkeit zwischen Philosophie und Gesellschaftstheorie. In: Conradi, Elisabeth / Vosman, Frans (Hrsg.): Praxis der Achtsamkeit. Schlüsselbegriffe der Care-Ethik. Frankfurt/New York, 54-86.

Diendorfer, Gertraud et. al. (Hrsg.) (2016): Friedensforschung, Konfliktforschung, Demokratieforschung. Ein Handbuch. Köln/Weimar/Wien.

District Berlin: Streitbauten. (2017). www.district-berlin.com/de/streitbauten/ (15.07.2020).

District Berlin: KLIRRRRR festival - queere Konfliktkulturen (2018). www.districtberlin.com/de/klirrrrr-ein-festival-queerer-konfliktkulturen/ (15.07.2020).

Institut für Queer Theory (o. J.): Queere Konfliktkulturen. www.queer-institut.de/ queere-konfliktkulturen/ (15.07.2020).

Laufenberg, Mike: Communities of Care. Queere Politiken der Reproduction. (2012). http://www. zeitschrift-luxemburg. de/communities-of-care-queere-politiken-derreproduktion (15.07.2020).

Lang, Colin: Common Ground. Colin Lang im Gespräch mit Julia Grosse, Suza Husse und Max Czollek. In: Texte zur Kunst 113 (2019). www.textezurkunst.de/113/113common-ground-de/ (15.07.2020).

Lind, Maria (2009): Complications. On Collaboration, Agency and Contemporary Art. In: Möntmann, Nina (Hrsg.): New Communities. Toronto. 
Manalansan IV, Martin F. (2018): Messy mismeasures. Exploring the wilderness of queer migrant lives. South Atlantic Quarterly 117(3), 491-506.

Povinelli, Elizabeth (2011): The Part That Has No Part. Enjoyment, Law, and Loss. In: GLQ. Gay and Lesbian Quarterly 17(2/3), 287-308.

Puig de la Bellacasa, Maria (2011): Matters of Care in Technoscience. Assembling Neglected Things. In: Social Studies of Science 41(1), 85-106.

Reitmair-Juárez, Susanne (2016): Entwicklungen, Schwerpunkte und Methoden der Friedenspädagogik. In: Gertraud Diendorfer et. al. (Hrsg.): Friedensforschung, Konfliktforschung, Demokratieforschung. Ein Handbuch. Köln / Weimar/Wien, 180-216.

Tobin, Amy (2019): Heresies' Heresies. Collaboration and Dispute in a Feminist Publication on Art and Politics. In: Women. A Cultural Review 30 (3), 280-296.

Tronto, Joan C. (2013): Caring Democracy. Markets, Equality, and Justice. New York.

Walgenbach, Katharina (2017): Heterogenität - Intersektionalität - Diversity in der Erziehungswissenschaft. Opladen. 